\title{
Symmetry Restoration in Gauge Boson Wave Packet Collisions in Yang-Mills-Higgs Theory
}

\author{
C. R. Hu, S. G. Matinyan, ${ }^{*}$ B. Müller \\ Department of Physics, Duke University \\ Durham, North Carolina 27ro8-0305
}

\begin{abstract}
We investigate the restoration of spontaneously broken gauge symmetry in collisions of gauge boson wave packets in the SU(2) Higgs model.
\end{abstract}

11.15.Kc, 11.30.Qc, 02.60.Cb

\section{INTRODUCTION}

The idea of the restoration of spontaneously broken gauge symmetries under extreme conditions (high temperature, high fermion densities, strong external gauge fields) was intensely studied in the 1970's and is well known [1].2] (for review see [3, (4) ). Under these conditions spontaneously broken gauge theories undergo various phase transitions resulting, in most cases, in the restoration of the originally broken symmetries. The consequences of such restorationmasslessness of the gauge bosons and fermions, long range character of the interactions, etc.- - are very important for the properties of the very early Universe. These properties may also play a significant role in the context of neutron stars and in the multiparticle production at high-energy interactions.

It has long been realized [1,2] that the Lagrangian of the Standard Model with a non-zero vacuum expectation value for the scalar field is a covariant generalization of the Ginzburg-Landau equation which describes a wide variety of "ordered" systems (ferromagnets, superfluids, superconductors, crystals, etc.) and their phase transitions. From this point of view very different systems have common general properties due to their differently revealed broken symmetries. Using the analogy with superconductivity, where a strong external electromagnetic current (magnetic field) destroys superconductivity resulting in the transition to the normal state, an intense current of weakly interacting particles (e.g. of $W$-bosons) can restore the gauge symmetry and cause the transition to a new phase with massless gauge quanta [2,5].

The present paper is devoted to the study of the restoration of the spontaneously broken gauge symmetry in the collisions of intense, energetic $W$-boson wave packets. The paper is organized as follows. Section II describes the symmetry restoration at zero temperature in the SU(2) Higgs model. In Section III this phenomenon is compared with the well-known results of symmetry restoration at high temperature. Section IV is devoted to the stability problem of the one-loop radiatively corrected effective potential with the external gauge field in the light of the existence of the heavy t-quark. In Section $\mathrm{V}$ we present lattice calculations of gauge boson wave packet collisions in which the restoration of the $\mathrm{SU}(2)$ gauge symmetry is manifested. Section VI contains short concluding remarks.

\section{RESTORATION OF THE BROKEN SU(2) SYMMETRY BY AN INTENSE GAUGE FIELD}

This paper is an outgrowth of previous studies of the interactions of gauge field wave packets in the SU(2) YangMills [6] and Yang-Mills-Higgs [7 theories. Being in essense classical, the approach of these publications nonetheless reveal interesting phenomena associated with the non-perturbative description of multiparticle production in collisions of weakly interacting particles. However, in the present study of symmetry restoration of the scalar field effective potential as a result of such wave packet collisions, this treatment is more adequate since the colliding of intense, energetic $W$-boson wave packets serve here as a space-time averaged external source exciting the vacuum of the Yang-Mills-Higgs system.

Our consideration is based on the spontaneously broken SU(2) Yang-Mills theory with one Higgs isodoublet coupled to the gauge field. This model retains the most relevant ingredients of the electroweak theory. We begin with some preliminaries.

The action describing the model in $(3+1)$ dimensions is given by

*Also at Yerevan Physics Institute, Yerevan, Armenia 


$$
S=\int d^{3} x d t\left[-\frac{1}{2} \operatorname{tr}\left(F_{\mu \nu} F^{\mu \nu}\right)+\frac{1}{2} \operatorname{tr}\left(\left(\mathcal{D}_{\mu} \Phi\right)^{\dagger} \mathcal{D}^{\mu} \Phi\right)-\lambda\left(\frac{1}{2} \operatorname{tr}\left(\Phi^{\dagger} \Phi\right)-v^{2}\right)^{2}\right],
$$

with $\mathcal{D}_{\mu}=\partial_{\mu}-i g A_{\mu}^{a} \tau^{a} / 2, \quad F_{\mu \nu} \equiv F_{\mu \nu}^{a} \tau^{a} / 2=(i / g)\left[\mathcal{D}_{\mu}, \mathcal{D}_{\nu}\right]$, and

$$
\Phi=\phi^{0}-i \tau^{a} \phi^{a},
$$

where $\tau^{a}(a=1,2,3)$ are Pauli matrices. We work in the unitary gauge where only physical excitations appear:

$$
\begin{aligned}
\Phi & =(v+\rho / \sqrt{2}) U(\theta), \\
A_{\mu} & =U(\theta) W_{\mu} U^{-1}(\theta)-\frac{1}{i g}\left(\partial_{\mu} U(\theta)\right) U^{-1}(\theta),
\end{aligned}
$$

with $U(\theta)=\exp \left(i \tau^{a} \theta^{a}\right)$ being a local gauge rotation.

The real field $\rho$ describes the oscillations of the scalar field about its vacuum expectation value $v$, and $W_{\mu}$ is the field of the gauge boson. $W_{\mu}$ and $\rho$ obey the following classical equations of motion:

$$
\begin{array}{r}
{\left[\mathcal{D}_{\mu}, F^{\mu \nu}\right]+M_{W}^{2} W^{\nu}+\frac{1}{\sqrt{2}} g^{2} v \rho W^{\nu}+\frac{1}{4} g^{2} \rho^{2} W^{\nu}=0,} \\
\left(\partial_{\mu} \partial^{\mu}+M_{H}^{2}\right) \rho+3 \sqrt{2} \lambda v \rho^{2}+\lambda \rho^{3}-\frac{\sqrt{2}}{4} g^{2} v W_{\mu}^{a} W^{a \mu}-\frac{1}{4} g^{2} \rho W_{\mu}^{a} W^{a \mu}=0 .
\end{array}
$$

$\mathcal{D}_{\mu}$ and $F^{\mu \nu}$ are defined in terms of $W^{\mu} . M_{H}=2 v \sqrt{\lambda}$ and $M_{W}=g v / \sqrt{2}$ are the masses of Higgs and gauge bosons, respectively.19

It is easy to see that after a scaling transformation

$$
x_{\mu}^{\prime}=g v x_{\mu}, \quad \Phi^{\prime}=\Phi / v, \quad A_{\mu}^{\prime}=A_{\mu} / v,
$$

the action (11) and the equations of motion (4) and (5) possess only a single parameter $\lambda^{\prime} \equiv \lambda / g^{2}=M_{H}^{2} / 8 M_{W}^{2}$. However, since in the following simulation of the colliding wave packets there are other parameters involved in the initial conditions, we consider the unscaled equations (4) and (5) in more detail.

Equation (5) describes the excitations of the scalar field about the Higgs vacuum $|\Phi|=v$. It has a second exact solution $\rho=-\sqrt{2} v$ (corresponding to $|\Phi|=0$ ) with $W_{\mu}^{a}$ being arbitrary. For this solution the last three terms in (ब) cancel each other and eq. (4) describes massless gauge bosons.

In terms of excitations $\chi=\rho+v \sqrt{2}$ around $|\Phi|=0$, the equations (雨) and (5) assume the form:

$$
\begin{aligned}
{\left[\mathcal{D}_{\mu}, F^{\mu \nu}\right]+\frac{1}{4} g^{2} \chi^{2} W^{\nu} } & =0, \\
{\left[\partial_{\mu} \partial^{\mu}-\frac{M_{H}^{2}}{2}\left(1+\frac{g^{2} W^{2}}{8 \lambda v^{2}}\right)\right] \chi+\lambda \chi^{3} } & =0 .
\end{aligned}
$$

The expression $g^{2} W^{2} / 8 \lambda v^{2}$ in (8), where $W^{2}(x) \equiv W_{\mu}^{a} W^{a \mu}$, plays an important role: Depending on the sign and magnitude of $W^{2}(x)$, the new "ground" state $|\Phi|=0$ can be unstable or stable. For positive $W^{2}(x)$ this state is obviously unstable noting that the effective mass of the scalar excitations described by (8) is imaginary.

For time-like $\left(k^{2}>0\right)$ gauge bosons $W^{2}(x)$ is always negative. In particular, this is so for transverse gauge bosons for which $W_{0}^{a}=0$. For space-like $\left(k^{2}<0\right)$ gauge bosons $W^{2}(x)$ can be of both signs. On the other hand, the luminosity of transversely polarized gauge bosons in proton-proton collisions at high energy, under realistic conditions, is much higher than that of longitudinally polarized ones and increases with energy [8]. Of course, the general suppression by a factor $M_{W} / E$ of the emission amplitude of longitudinal $W$-bosons from a quasi-massless fermion is compensated by the nonvanishing of this amplitude in the forward direction in contrast to that for transverse $W$-bosons leading, in general, to the dominance of the cross-sections for longitudinal $W$ - $W$ interactions over transverse ones [8]. But, as

\footnotetext{
${ }^{1}$ Note that the gauge field acts as a source for Higgs field excitations in (5). This permits us to consider the $W$-field classically with respect to the excitation of the scalar field. The equation (4) for gauge field does not possess a source term.

${ }^{2}$ Our normalization of the $\rho$-oscillations about $v$ in (3) corresponds to the choice $v=174 \mathrm{GeV}$. Thus, $G_{F} / \sqrt{2}=1 / 4 v^{2}$, where $G_{F}$ is the effective four-fermion coupling constant.
} 
seen from the above considerations, for the restoration of symmetry the important factor is not the cross section of $W-W$ interaction, but rather the intensity of the "pulse" $W^{2}$ which appears in equation (8) as an external source (cf. the footnote 1).

Concerning the $W-W$ collisions, in the following we will consider collisions of transverse gauge bosons for which the relation $\partial^{\mu} W_{\mu}^{a}=0$ holds. This also allows us to work in the temporal gauge $W_{0}^{a}=0$, which is convenient for lattice calculations in the Hamiltonian formulation (see Section $\mathrm{V}$ for details). We would like to emphasize that, considering the case of transversely polarized $W-W$ scattering, we exclude the important issue of the potentially strong longitudinal $W-W$ interaction which may exist beyond the Standard Model [9]. The restoration of the broken symmetry in the transverse $W-W$ collisions studied here is a phenomenon entirely within the confines of the Standard Model.

Since the colliding wave packets are highly energetic $\left(\bar{k} \gg M_{W}, M_{H}\right)$ and rapidly oscillating in space and time, the Higgs field oscillations will only follow effectively the average of the gauge wave packets. We therefore introduce the space-time averaged quantity

$$
\eta \equiv \frac{g^{2}\left\langle\left(W_{i}^{a}\right)^{2}\right\rangle}{8 \lambda v^{2}}=\frac{M_{W}^{2}}{M_{H}^{2}} \frac{\left\langle\left(W_{i}^{a}\right)^{2}\right\rangle}{v^{2}} .
$$

Equation (8) describes the excitations of the scalar field $\chi$ with an effective mass

$$
\tilde{M}_{H}^{2}=\frac{M_{H}^{2}}{2}(\eta-1) .
$$

For $\eta<1$ the excitations around the $\Phi=0$ ground state is tachyonic, indicating instability. For $\eta>1$, however, these oscillations are stable. In particular, for the spatially homogeneous case there exists an exact stable solution of (\&)

$$
\chi(t)=\frac{\chi_{0} e^{i \tilde{M}_{H} t}}{1-\frac{\lambda}{8 \tilde{M}_{H}^{2}} \chi_{0}^{2} e^{2 i \tilde{M}_{H} t}},
$$

where $\chi_{0}$ is a constant $[10]$.

It is instructive to analyze the effective potential $V(\chi, \eta)$ for the excitations $\chi$ described in equation (8):

$$
V(\chi, \eta)=-\lambda v^{2}(1-\eta) \chi^{2}+\frac{\lambda}{4} \chi^{4}
$$

Equation (12) has two different stable minima, depending on the magnitude of $\eta$ :

$$
\begin{aligned}
& \text { For } \eta<1, \quad \chi_{\min }= \pm \sqrt{2} v(1-\eta)^{1 / 2}, \quad \text { i.e. }|\Phi|=v(1-\eta)^{1 / 2} . \\
& \text { For } \eta \geq 1, \quad \chi_{\min }=0, \quad \text { i.e. } \Phi=0 .
\end{aligned}
$$

Stable excitations about these "vacua" have the following squared masses:

$$
\begin{aligned}
& \tilde{M}_{H}^{2}=\frac{M_{H}^{2}}{2}|1-\eta|[1+\theta(1-\eta)], \\
& \tilde{M}_{W}^{2}=M_{W}^{2}(1-\eta) \theta(1-\eta) .
\end{aligned}
$$

Thus for $\eta>1$, the broken $\mathrm{SU}(2)$ gauge symmetry is restored and oscillations of the scalar field occur about the symmetrical state $|\Phi|=0$, not about $|\Phi|=v$. The effective mass of the gauge bosons in the region where the symmetry is restored $(\eta>1)$ vanishes. For $\eta<1$, the vacuum is changed gradually as $(1-\eta)^{\frac{1}{2}}$, approaching $|\Phi|=0$. For $\eta<1$, the ratio $r=\tilde{M}_{H} / \tilde{M}_{W}=M_{H} / M_{W}$ has no dependence on $\eta$.

\section{COMPARISON WITH THE HIGH TEMPERATURE CASE}

The above described features are characteristics of a second order phase transition. From this point of view, it is illuminating to compare the restoration of symmetry in an intense gauge field associated with time-like $W$-bosons $\left(k^{2}>0\right.$, or $\left.W_{\mu}^{a} W^{a \mu}<0\right)$ with the analogous phenomenon occurring at high temperature.

For the same SU(2) Higgs model the parameter governing the restoration of symmetry at finite temperature at one-loop level is 111 . 13 


$$
\eta_{T}=\frac{T^{2}}{v^{2}}\left(\frac{1}{3}+\frac{M_{W}^{2}}{M_{H}^{2}}\right)
$$

The high temperature acts here in the same way as an intense time-like gauge field (or as an extra-high fermion current density [3, 4 ). The joint action of these two effects, of course, would make the transition easier. It is important to stress that our essentially classical approach is not reliable for $\eta$ close to unity where the radiative corrections are significant even at small coupling constants. However, it is expected that the radiative corrections are not important in the region described by the inequality [3].

$$
|1-\eta| \geq \lambda, g^{2}
$$

\section{ON THE ROLE OF THE RADIATIVE CORRECTIONS AT ONE LOOP LEVEL}

Evidence of the existence of the very heavy $t$-quark $\left(m_{t} \approx 175 \mathrm{GeV}\right)$ [14 raises the question of the stability of the ground state with and without external influence. In this section we show that the possible inclusion of heavy fermions into our radiatively corrected effectived potential (12), under realistic conditions outlined in Section 3, does not change the resulting picture for moderate $\eta$ and the perturbative range of $\lambda$ and $g^{2}$.

The radiatively corrected potential (12) has a well-known contribution from one loop diagrams including the physical $\mathrm{SU}(2)$ gauge bosons, Higgs scalar, and $t$-quark 15. (we ignore other much lighter quarks and other scalars). We have for our choice of the normalization of the $\chi$-excitations and of $v$ :

$$
V_{c}(\chi, \eta)=V(\chi, \eta)+\frac{C}{4} \chi^{4} \ln \frac{\chi^{2}}{2 M^{2}}
$$

where $M$ is an arbitrary normalization scale which will be chosen in the following equal to $v$. For $C$ we have ${ }^{3}$ :

$$
C=\frac{1}{256 \pi^{2} v^{4}}\left[\sum_{V} 3 M_{V}^{4}+M_{H}^{4}-4 M_{t}^{4}\right] .
$$

Inserting into (20) the physical masses of particles, we are left with

$$
C=4 \times 10^{-4}\left[\left(\frac{M_{H}}{v}\right)^{4}-3.5\right] .
$$

As we are interested in the possible destablizing role of the $t$-quark, we consider the case $C<0$, i.e. $M_{H}<238 \mathrm{GeV}$, or, $\lambda<0.47$. On the other hand, $\lambda>0.03$ follows from the present experimental lower bound on the Higgs mass $\left(M_{H}>65.2 \mathrm{GeV}\right)[16$.

Now, the minimum of the full effective potential $(19)$ is

$$
\chi_{\min }^{2}=\frac{2 v^{2}(1-\eta)}{1+\frac{C}{\lambda}\left[\ln \frac{v^{2}(1-\eta)}{M^{2}}+\frac{1}{2}\right]} \cdot \theta(1-\eta) .
$$

The term $\frac{1}{2}$ in the denominator of (22), of course, can be absorbed into $M$. The prefactor of the logarithm in (22) is small $\left(\frac{|C|}{\lambda}<0.05\right)$.

The condition for the validity of the one-loop approximation (we take $M \sim v$ ) is:

$$
\begin{array}{ll}
1-\eta>e^{-\frac{\lambda}{|C|}}, & \text { for } \eta<1 \\
\eta-1<e^{\lambda /|C|}, & \text { for } \eta>1
\end{array}
$$

\footnotetext{
${ }^{3}$ The unconventional numerical factor in $C$ is due to our normalization of the $\rho$-oscillations in (3) and our choice of $v=174$ $\mathrm{GeV}$. Summation in (20) runs over gauge bosons $W^{ \pm}$and $Z$.
} 
which is well satisfied considering our restriction (18) of the whole reliability of the approach and for not very large $\eta$. Thus we conclude that the picture does not change drastically by the inclusion of the radiative corrections with the dominated $t$-quark contribution.

Indeed, one has for $|\eta-1|>\lambda, g^{2}$ and not large $\eta$ :

$$
\begin{aligned}
|\Phi|_{\min } & =v(1-\eta)^{1 / 2}\left[1-\frac{C}{2 \lambda} \ln (1-\eta)\right] \theta(1-\eta), \\
\tilde{M}_{H}^{2} & =\frac{M_{H}^{2}}{2}|1-\eta|\left(1-\frac{C}{\lambda}\right)(1+\theta(1-\eta)), \\
\tilde{M}_{W}^{2} & =M_{W}^{2}(1-\eta)\left(1-\frac{C}{\lambda} \ln (1-\eta)\right) \theta(1-\eta), \\
\lambda_{c} & \left.\equiv \frac{1}{6} \frac{d^{4} V}{d \chi^{4}}\right|_{\chi=\chi_{\min }}=\lambda\left[1+\frac{C}{\lambda}\left(\ln |1-\eta|+\frac{23}{6}\right)\right] .
\end{aligned}
$$

From (25) it is seen that the use of the bare coupling constant $\lambda$ in all previous estimates is reasonable.

The physical reason for this small influence of the $t$-quark is clear: the choice of moderate external vector field amplitude $W^{2}(<0)$ ensures the reliability of one-loop approximation and, as we see, the radiative corrections cannot stop the tendency towards the restoration of the symmetry.

It is worthy to emphasize that whereas our arguments are well grounded for the $\eta<1$ region, relations (25) have only qualitiative validity even for moderate $\eta$ in the region $\eta>1$ where infrared singularities connected with the masslessness of the gauge bosons are important and practically incurable. These difficulties are common for phase transitions in many-body problems treated by perturbation theory or by mean field methods [3].

Note, finally, that in the potential (19) the renormalization scale dependence of its parameters is ignored. However, the one-loop potential (19) has all the qualitative features of the "full" renormalization group improved potential, and the results thus obtained will not be substantially changed if we use the "exact" potential [17].

\section{RESTORATION OF THE SU(2) GAUGE SYMMETRY IN GAUGE WAVE PACKET COLLISIONS}

As follows from the arguments in Section IV, the reliability of our analysis is confined to the values of not very large $\eta$. For $\eta \gg 1$ our treatment is, in principle, not well grounded, but that occurs only in the very early Universe. On the other hand, the study of the gradual decrease of the vacuum expectation value as a function of $\eta$ from $|\Phi|=v$ to $|\Phi|=v(1-\eta)^{1 / 2}$ for $\eta<1$ is free from the difficulties mentioned in Section IV.

In this section we study the collision of gauge wave packets from the point of view of the restoration of the $\mathrm{SU}(2)$ broken symmetry. In [7] we have investigated the evolution of the gauge and Higgs's field as a function of the colliding wave packet amplitudes and of the mass ratio $r \equiv M_{H} / W_{W}$. For a wide range of the essential parameters we have observed collisions of the initial wave packets resulting in final configurations with dramatically different momentum distributions. Note that the classical approach used in these calculations, although suffering from the common ultraviolet catastrophe of classical field theory revealed in the momentum distribution of the final states (see [7]), is adequate in the present context since we are not interested in the final states.

Our numerical study here, as in [7], is based on the Hamiltonian formulation of lattice gauge theory [18] (see [19, [6] for more details), in which the dynamical variables are link variables defined as

$$
U_{\ell}=\exp \left(-i g a A_{\ell}^{c} \tau^{c} / 2\right)
$$

( $\ell$ is the link index.) As in [6,7] we work with a one-dimensional lattice of size $L=N a$, where $N$ is the number of lattice sites and $a$ the lattice spacing. As mentioned in Section II, we "collide" transverse $W$-bosons to ensure the negativity of $W_{\mu}^{a} W^{a \mu}$ as well as the temporal gauge condition $W_{0}^{a}=0$.

The initial configuration is given by two well-separated left- and right-moving wave packets originally centered at $z_{L(R)}$ with average momenta $k=(0,0, \mathbf{k})$ and width $\Delta k . z_{L(R)}$ are chosen so that the wave packets are positioned symmetrically about the center of the lattice:

$$
\begin{aligned}
& W^{c, \mu}=W_{\mathrm{L}}^{c, \mu}+W_{\mathrm{R}}^{c, \mu}, \\
& W_{\mathrm{L}}^{c, \mu}=(0,0,1,0) n_{\mathrm{L}}^{c} \psi\left(z-z_{\mathrm{L}},-t\right), \\
& W_{\mathrm{R}}^{c, \mu}=(0,0,1,0) n_{\mathrm{R}}^{c} \psi\left(z-z_{\mathrm{R}}, t\right),
\end{aligned}
$$

with $n_{\mathrm{L}}^{c}$ and $n_{\mathrm{R}}^{c}$ being the polarization vectors in isospin space. 
A right-moving wave packet centered at $z=0$ at $t=0$ with mean wave number $\bar{k}$, mean frequency $\bar{\omega}=\left(\bar{k}^{2}+M_{W}^{2}\right)^{1 / 2}$ and width $\Delta k$ is described by

$$
\psi(z, t)=\frac{\hbar^{1 / 2}}{\sqrt{\pi^{3 / 2} \Omega \Delta k \sigma}} \int_{-\infty}^{+\infty} d k_{z} e^{-\left(k_{z}-\bar{k}\right)^{2} / 2(\Delta k)^{2}}\left[\cos \left(\omega t-k_{z} z\right)\right]
$$

with $\omega=\sqrt{k_{z}^{2}+M_{W}^{2}}$, and

$$
\Omega=\bar{\omega}\left[1+\frac{1}{4}\left(1-\left(\frac{\bar{k}}{\bar{\omega}}\right)^{2}\right)\left(\frac{\Delta k}{\bar{\omega}}\right)^{2}+O\left(\frac{\Delta k}{\bar{\omega}}\right)^{4}\right]
$$

where the amplitude is fixed by requiring energy equal to $\hbar \bar{\omega}$ per cross-sectional area $\sigma$. (In the following we set $\hbar=1$.) From (28) we obtain

$$
\left.\psi\right|_{t=0}=\sqrt{\frac{2 \Delta k}{\sqrt{\pi} \Omega \sigma}} e^{-(\Delta k z)^{2} / 2} \cos (\bar{k} z)
$$

and

$$
\left.\frac{d}{d t} \psi\right|_{t=0}=\sqrt{\frac{2 \bar{\omega} \Delta k}{\sqrt{\pi} \sigma(\Omega / \bar{\omega})}} e^{-(\Delta k z)^{2} / 2}\left[\sin (\bar{k} z)+\frac{\bar{k}}{\bar{\omega}} \frac{\Delta k}{\bar{\omega}} \Delta k z \cos (\bar{k} z)+\mathcal{O}\left(\left(\frac{\Delta k}{\bar{\omega}}\right)^{2}\right)\right] .
$$

Initial condition for the Higgs field is the vacuum solution:

$$
\phi^{0}=v, \quad \phi^{a}=0, \quad \dot{\phi}^{0}=\dot{\phi}^{a}=0 \quad \text { at } \quad t=0 .
$$

To determine the number of independent parameters, it is useful to rescale (30) according to (6) in the high-energy limit $(\Omega \approx \bar{\omega} \approx \bar{k})$ :

$$
\left.\psi^{\prime}\right|_{t^{\prime}=0}=\left.\frac{1}{v} \psi\right|_{t=0} \approx \sqrt{\frac{2 \Delta k}{\sqrt{\pi} \bar{k} \sigma v^{2}}} e^{-\left(\frac{\Delta k}{g v} z^{\prime}\right)^{2} / 2} \cos \left(\frac{\bar{k}}{g v} z^{\prime}\right)
$$

which contains three dimensionless parameters: $\bar{k} / M_{W}, \Delta \bar{k} / M_{W}$, and $\sigma M_{W}^{2} / g[7]$. There appears one more parameter in the initial conditions - the relative initial isospin orientation of the colliding wave packets, $\theta_{c}$. As shown in [6, ]], the parameter $\theta_{c}$ plays an important role in the collisions: Whereas for $\theta_{c} \neq 0$ (essentially non-abelian configuration) collisions of the wave packets resulted in highly inelastic final states, for parallel isospins (pure abelian configuration) the collisions were practically elastic. However, for the phenomenon we are interested in here - restoration of the broken symmetry - the coupling between the gauge and Higgs field is essential and not the relative orientation of the gauge bosons in isospin space.

It is convenient to have a scale parameter in the dimensionless parameters $v$ rather than $M_{W}$. Combining equations of motion and initial conditions, we finally have four independent parameters:

$$
r \equiv \frac{M_{H}}{M_{W}}, \frac{\bar{k}}{v}, \frac{\Delta k}{v}, \text { and } \frac{\sigma v^{2}}{g^{2}} .
$$

In our simulations we always chose $\bar{k} \gg M_{W}$ and $\bar{k} \gg \Delta k$ to model high energy collision and to have wave packets as well-defined objects. The gauge coupling constant was fixed to $g=0.65$.

We estimated the parameter $\eta$ using two different configurations of the gauge boson wave packets. Firstly, we consider the configuration of the two incident wave packets at the space-time point when they overlap completely. Then from (9), (27), and (28) we have

$$
\eta= \begin{cases}\frac{4}{r^{2}} \kappa^{2}, & \text { for parallel isospin orientation } \\ \frac{2}{r^{2}} \kappa^{2}, & \text { for orthogonal isospin orientation } \\ 0, & \text { for antiparallel orientation }\end{cases}
$$

where $\kappa=\frac{2 \Delta k}{\sqrt{\pi} k \sigma v^{2}}$ is just the parameter we have introduced earlier (see (32) in [6]). As found there, for $\kappa \geq 1$ the $W-W$ wave packet collisions produced strongly inelastic final states (for non-parallel isospin orientation). However, one should note that according to Eq. (5) the scalar field reacts to the gauge field long before the overlapping of 
the wave packets (see also figures below). That makes the behavior of the collisions less sensitive to the relative orientations of the wave packets in isospin space. Practically, as seen in our simulations, there is no difference between the parallel and the antiparallel cases.

Let us estimate $\eta$ taking the initial $(t=0)$ configurations of two separate wave packets. From (9), (28), and (34) we have after spatial averaging

$$
\eta=\frac{\kappa^{\prime 2}}{r^{2}} \equiv \frac{2 \Delta k}{\bar{k} \sigma v^{2} r^{2}} .
$$

Note that no isospin factor appears here after spatial averaging. As we see, the two estimates (35) and (36) almost coincide. A sufficiently large $\eta$ is achievable for realistic values of $r>1$ and permissible ratio $\Delta k / \bar{k}$ if we take $\sigma<1 / v^{2}$.

The natural spread of the $W$-boson wave packets emitted by fast moving fermions (quarks, leptons) is of order $v$ in the comoving reference frame. Therefore, the transverse area of the $W$-boson wave packet is not larger than $1 / v^{2}$. Of course, the more reliable estimate of $\eta$ depends on the detailed shape of the wave packet and requires a full three-dimensional analysis.

Figures 1 and 2 describe the results of our lattice simulations of the collisions of $W$ wave packets. In view of the above mentioned relation between the parameters $\eta$ and $\kappa$ (or $\kappa^{\prime}$ ) (see (35) and (36)) we present here only results for wave packets with orthogonal isospins since parallel isospin orientation leads qualitatively to the same symmetry restoration patterns.

In the first column of Figure 1, the space development of the colliding $W$ wave packets with $\eta=1.32$ is shown for different times. As seen from this figure, at time $t \approx 300$ the $W$ wave packets collide and then begin to separate. Just about at this time one expects to observe the restoration of symmetry, i.e. the oscillations of the scalar field $|\Phi|$ about a new ground state located below the "old" vacuum $|\Phi| / v=1$. After the separation of the wave packets $(t>300)$ the excitations of the scalar field tend again to oscillate about the "old" vacuum, i.e. the gauge symmetry is broken again. This is seen clearly in the second and third columns of Figure 1. The second column shows the space-time evolution of the magnitude of the Higgs field $|\Phi| / v$. The third column shows the Higgs field smoothed over 50 lattice sites, suppressing the small scale fluctuations, in order to facilitate a comparison with the definition (9) of the effective parameter $\eta$ in terms of the averaged strength of the $W$-boson field. At the moment of interaction, $t \approx 300$, the lowering of the average value of the Higgs field at the location of the coalescing wave packets is clearly visible.

In Figure 2, we show the $\eta$ dependence of the $W-W$ collisions (first column) and the corresponding behavior of the scalar field (second and third columns) at $t=300$. To change $\eta$ we vary the amplitude of wave packets by changing $\sigma$; all other parameters are kept fixed. It is evident that no significant symmetry restoration occurs for $\eta<1$, as expected. For $\eta \gg 1$ the situation becomes more complicated, probably because already a single wave packet can restore the symmetry in this case. It is seen, however, that the spatial region with restored symmetry becomes wider as the parameter $\eta$ grows.

\section{CONCLUDING REMARKS}

The above study of colliding gauge wave packets in the spontaneously broken SU(2) Yang-Mills-Higgs model visualizes the process of the $\mathrm{SU}(2)$ gauge symmetry restoration in some finite space-time region as a result of the presence of the intense gauge pulses. In some sense, it qualitatively resembles the situation realized in the very early Universe with the Big Bang scenario where the restoration is caused by the high temperature. The approach used in this paper might be applied to the study of chiral symmetry restoration. As is well-known, there exists an isomorphism between the Higgs sector of the standard model and the linear $\sigma$-model. This isomorphism is realized through the following identifications: $W_{L} \leftrightarrow \pi, H \leftrightarrow \sigma, v \leftrightarrow f_{\pi}$, where $W_{L}$ is the longitudinal gauge field and $H$ the physical Higgs field. $f_{\pi}$ is the $\pi$-decay constant defining the scale of the chiral dynamics. As a consequence of this isomorphism, one might expect that the spontaneously broken chiral symmetry can also be restored in the presence of a strong external pion field and such a physical setting might be found in heavy-ion collisions or in the interior of nuclei and neutron stars.

We have seen in Section $\mathrm{V}$ that the restoration of the gauge symmetry in $W$-wave packet collisions occurs at amplitudes for which strongly inelastic behavior has also been observed. To achieve a sufficient value of the amplitude is not easy in practice. The problem, presumably, is connected with the general problem of the classical (quasiclassical) treatment of the amplitude for the transition from a few-particle to a many-particle final state (see, e.g. [20]). If, as conjectured in [21] (see also 22 24]), there exist classical trajectories interpolating between incoming few-particle and outgoing multi-particle states, our results would lead us to expect that these trajectories will also exhibit the phenomenon of symmetry restoration in the transition region. It is also worthwhile mentioning that the classical approach is more adequate in the context discussed here, where the colliding intense, energetic $W$-boson 
wave packets serve as a classical external source exciting the ground state of the Yang-Mills-Higgs system, and we are not interested in the specific nature of the final states.

In conclusion, we emphasize that the restoration of the gauge symmetry discussed above does not depend on the dimensionality of space. It is expected that the symmetry restoration would not persist as long in three space dimensions because the wave packets disperse more rapidly after the collisions, causing the average squared amplitude $\left\langle\left(W_{i}^{a}\right)^{2}\right\rangle$ to decay more rapidly. The same arguments can apply to the problem of multiparticle production in wave packet collisions [6]:7]. This issue, as well as the problem of the reliability of the classical treatment of the (few $\rightarrow$ many) particle amplitude makes the study of the gauge wave packet collisions in $(3+1)$ dimensions desirable.

\section{ACKNOWLEDGMENTS}

We thank K. Rajagopal and R. Singleton for fruitful discussions and comments. This work was supported, in part, by grant DE-FG02-96ER40945 from the U. S. Department of Energy, and by the North Carolina Supercomputing Center.

[1] D. A. Kirzhnits, JETP Lett. 15, 529 (1972).

[2] D. A. Kirzhnits and A. D. Linde, Phys. Lett. 42B, 471 (1972).

[3] D. A. Kirzhnits and A. D. Linde, Ann. Phys. (NY) 101, 195 (1976).

[4] A. D. Linde, Rep. Prog. Phys. 42, 389 (1979).

[5] I. V. Krive, V. M. Pyzh, and E. M. Chudnovskii, Sov. J. Nucl. Phys. 23, 258 (1976).

[6] C. R. Hu, S. G. Matinyan, B. Müller, A. Trayanov, T. M. Gould, S. D. Hsu, and E. Poppitz, Phys. Rev. D52, 2402 (1995).

[7] C. R. Hu, S. G. Matinyan, B. Müller and D. Sweet, DUKE-TH-95-97, to appear in Phys. Rev. D.

[8] M. S. Chanowitz and M. K. Gaillard, Phys. Lett. B142, 85 (1984); S. Dawson, Nucl. Phys. B249, 42 (1985).

[9] J. M. Cornwall, D. N. Levin, and G. Tiktopoulos, Phys. Rev. D10, 1145 (1974); B. W. Lee, C. Quigg, and M. B. Thacker, Phys. Rev. D16, 1519 (1977); M. S. Chanowitz and M. K. Gaillard, Nucl. Phys. B261, 379 (1985).

[10] L. S. Brown, Phys. Rev. D46, R4125 (1992).

[11] S. Weinberg, Phys. Rev. D9, 3357 (1974).

[12] L. Dolan and R. Jackiw, Phys. Rev. D9, 3320 (1974).

[13] D. A. Kirzhnits and A. D. Linde, Sov. Phys. JETP 40, 628 (1975).

[14] F. Abe et al. CDF Collaboration, Phys. Rev. Lett. 74, 2626 (1995); Phys. Rev. D52, R2605 (1995); S. Abachi et al. D0 Collaboration, Phys. Rev. Lett. 74, 2632 (1955).

[15] S. Coleman and E. Weinberg, Phys. Rev. D7, 1888 (1973).

[16] J.-F. Grivaz, Proceedings of the Intern. EPS Conference on High-Energy Physics, Bruxelles, 1995.

[17] M. Sher, Physics Reports 179, 273 (1989).

[18] J. Kogut and L. Susskind, Phys. Rev. D11, 395 (1975).

[19] T. S. Biró, C. Gong, B. Müller, and A. Trayanov, Int. J. Mod. Phys. C5, 113 (1994).

[20] M. B. Voloshin, in Proceedings of the 27th International Conference on High Energy Physics, Glasgow, Scotland, 1994; v. 1, p. 121.

[21] V. Rubakov, D. Son, and P. Tinyakov, Phys. Lett. B287, 342 (1992).

[22] T. M. Gould, S. D. H. Hsu, and E. R. Poppitz, Nucl. Phys. B437, 83 (1995).

[23] C. Rebbi and R. Singleton, preprint <hep-ph/9601260 , Boston University (1996), to appear in Phys. Rev. D.

[24] E. Farhi, J. Goldstone, A. Lue, and K. Rajagopal, MIT preprint CTP - 2483, 1995. 

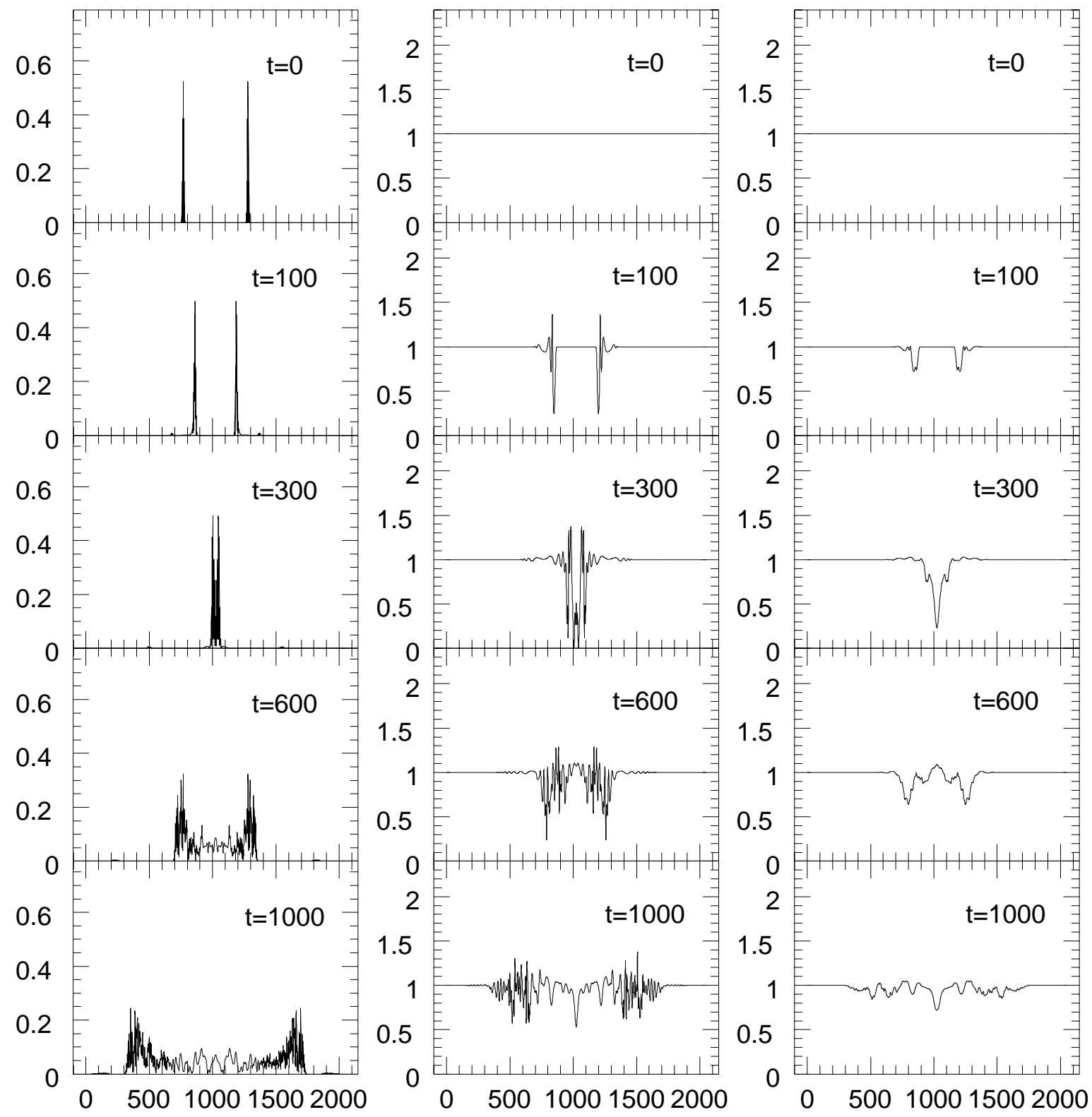

FIG. 1. Space-time development of symmetry restoration induced by two colliding gauge wave packets with orthogonal isospins in the presence of the Higgs vacuum condensate. First column shows the scaled gauge field $|\mathbf{A}| / v$ as a function of space coordinate $z$ at five chosen times. Second column demonstrates the corresponding space-time evolution of the scaled Higgs field $|\Phi| / v$. Third column shows the scaled Higgs field after smoothing over 50 lattice sites. This simulation as well as the following (Fig. 2) was done on a lattice of sites $N=2048$ and lattice spacing $a=1$. The parameters were $\bar{k}=\pi / 4, \Delta k=\pi / 16$, $M_{H}=M_{W}=0.15, g=0.65$, and $\sigma=1$. 

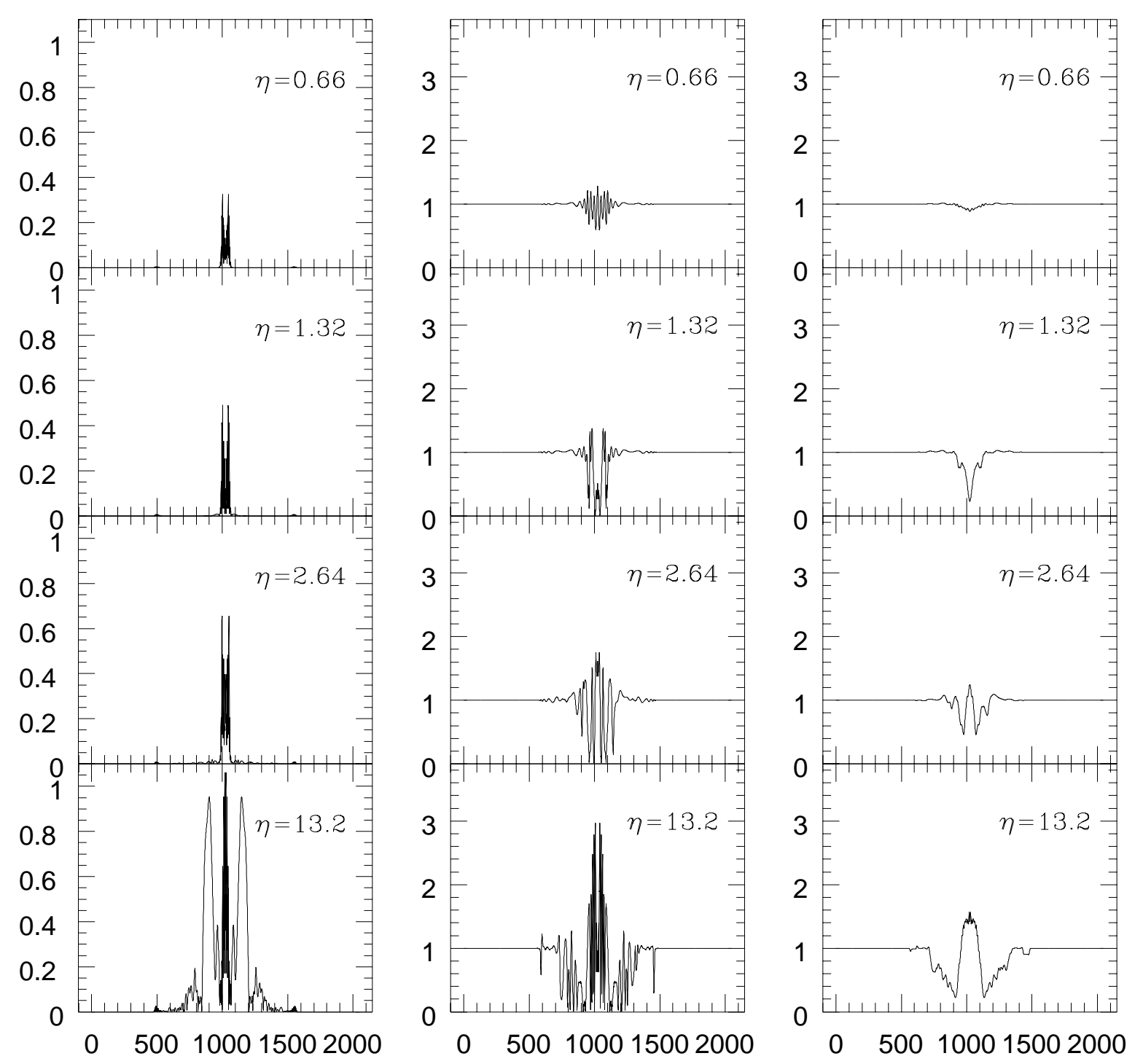

FIG. 2. $\eta$-dependence of the symmetry restoration. We demonstrate at a chosen time $t=300$ the space pictures of the colliding gauge wave packets and the Higgs field for four different values of $\eta$. First, second, and third columns shows the scaled gauge field $|\mathbf{A}| / v$, the scaled Higgs field $|\Phi| / v$, and the smoothed Higgs field $|\Phi| / v$, respectively, each as a function of the space coordinate $z$. All parameters were the same as in Fig. 1 except $\sigma$, which was to varied to arrive at different values of $\eta$. 